$$
\text { "fatalin" — 2005/6/30 - 16:51 — page } 1 \text { - \#1 }
$$

\title{
Über den Vergleich des mathematischen bzw. mathematikdidaktischen Vektorbegriffs durch den Galois-Graphen
}

\author{
LÁSZLÓ FATALIN
}

\begin{abstract}
In this article we show how to apply the method of Galois-graph - one of the means of the formal concept-analysis in order to coordinate the mathematical and didactical requirements. As an example we have chosen the concept of the "vector". As a result of the analysis it is proved that, in elaborating the right vector concept the geometric and algebraic foundations are both needed. The analysis also points out that the geometric model, based on the concept of the "directed segment" is unnecessarily overemphasized in the East-European education.
\end{abstract}

Key words and phrases: Vektorbegriff, formale Begriffsanalyse.

ZDM Subject Classification: C30, E40, G70.

Einen wichtigen Bereich in der Mathematikdidaktik stellt die Begriffsbildung dar (Ambrus, 2002). In diesem Artikel wird ein Ausbilden des Vektorbegriffs mit einem Mittel der formalen Begriffsanalyse, mit dem Galois-Graphen untersucht. Diese Untersuchung beruht auf einem elementaren Vektormodell und seinen Eigenschaften nach der Fachliteratur.

\section{Die Untersuchungsmethode}

Die formale Begriffsanalyse wurde von Garrett Birkhoff (1940) begründet und von der Darmstädter Gruppe um Rudolf Wille (1996) weiterentwickelt. Der 


$$
\text { "fatalin" - 2005/6/30 - 16:51 - page } 2-\# 2
$$

Ausgangspunkt der formalen Begriffsanalyse ist eine binäre Relation, d.h. ein geordnetes Tripel $(O, E, R)$ aus den folgenden Mengen: einer Objektmenge $O$, einer Eigenschaftsmenge $E$ und einer Relation $R \subseteq O \times E$ zwischen ihnen. (Die Schreibweise $(o, e) \in R$ wird gelesen als: das Objekt $o$ besitzt die Eigenschaft $e$.) Der Gegenstand der formalen Begriffsanalyse sind binäre Relationen und zu ihnen in enger Verbindung stehende vollständige Verbände. Die pädagogischen Anwendungen der formalen Begriffsanalyse wurden von Viola Takács (2000) untersucht. In den pädagogischen Anwendungen bekommen die Galois-Graphen der Untersuchungsrelation die Hauptrolle. Die Galois-Graphen können dann im Bereich der Anwendung zu neuen Einsichten verhelfen.

In mathematikdidaktischer Anwendung geht die formale Begriffsanalyse von einem Verhandlungskontext aus. Zuerst müssen eine Menge von Objekten und eine Menge von Untersuchungsaspekten abgegrenzt werden. Wenn die Analyse der Eigenschaften von Objekten in allen Fällen ein logisch eindeutiges Ergebnis (true/false) bringt, dann kann dieser Verhandlungskontext mit der Relation zwischen den Objekten und seinen Eigenschaften modelliert werden. Ein Verhandlungskontext wird meistens durch eine Kontexttabelle veranschaulicht, in der Kreuze den Elementen der Relation entsprechen. (Diese Kontexttabelle definiert, in welcher Beziehung eine Menge von Objekten zu einer Menge von Eigenschaften steht. Objekte werden darin per Konvention als Zeilen, Eigenschaft als Spalten dargestellt, siehe Tabelle 1.) Die Darstellung der Kontexttabelle (die Umgrenzung der Mengen und das Ausfüllen der Tabelle) verlangt gleichzeitig die mathematische und auch die mathematikdidaktische Kompetenz.

Die Cliquen sind die maximalen geschlossenen Teilmengen der Relation. Eine Teilmenge $C$ der Relation $R$ ist geschlossen, wenn eine Teilmenge $O^{\prime}$ der Objektmenge $O$ und eine Teilmenge $E^{\prime}$ der Eigenschaftsmenge $E$ existieren, so dass die Teilmenge $C$ als $C=O^{\prime} \times E^{\prime} \subseteq R$ geschrieben werden kann. (Jedes, in einer Clique enthaltene Objekt besitzt alle für das Clique charakteristische Eigenschaften). Eine geschlossene Teilmenge ist maximal, wenn sie sich weder durch Objekte noch durch Eigenschaften erweitern lässt. Eine Clique $C=O^{\prime} \times E^{\prime}$ kann als ein Modell für einen potentialen Begriff $B$ betrachtet werden, wobei $O^{\prime}$ den Begriffsumfang (die Begriffsbedeutung) und $E^{\prime}$ den Begriffsinhalt (den Begriffssinn) von $B$ modelliert.

Über der Menge der Cliquen ist eine Ordnungsrelation in folgender Weise einführbar: die Clique $C_{1}=O_{1} \times E_{1}$ ist mehr als eine Clique $C_{2}=O_{2} \times E_{2}$, wenn sich $O_{1} \supseteq O_{2}$ und $E_{1} \subseteq E_{2}$ im mengentheoretischen Sinne verwirklichen. Diese dualitäre Begriffsbeschreibung zeigt sich das Folgende: die Verallgemeinerung des 


$$
\text { "fatalin" - 2005/6/30 - 16:51 - page } 3-\# 3
$$

Begriffsumfangs bedeutet gleichzeitig die Spezialisierung des Begriffsinhalts. Auch umgekehrt ist dies lesbar: die Generalisation der Begriffsinhalte ergibt gleichzeitig die Spezialisierung der Begriffsumfänge.

Der Galois-Graph des Verhandlungskontexts ist das Hasse-Diagramm der geordneten Menge von Cliquen. Der Galois-Graph des Relationsmodells des Verhandlungskontexts gibt eine strukturelle Beschreibung von Begriffssystemen für mathematikdidaktische Konklusionen (Fatalin 2003a).

\section{Mathematikdidaktischen Überlegungen}

Der abstrakte Vektorbegriff wurde bereits Mitte des XIX. Jahrhundert begründet. Hamilton hat die algebraischen Gründe des Vektorbegriffs in der Theorie der Quaternionen und Grassmann die geometrische Deutung begründet. Nach einem Jahrhundert hat der Vektorbegriff auch seinen Ort im Lehrstoff der Mittelschule erhalten (Boltjanszkij \& Jaglom, 1963). Die Einführung dieses Begriffs wurde auch durch vielfältige Anwendungen motiviert; überdies ist es ein gutes Gebiet der abstrakten Denkentwicklung von der anschaulichen bis zur abstrakten Definition.

Die mathematikdidaktische Fachliteratur gliedert die auftauchenden Probleme auf Grund von Unterrichtserfahrungen in die folgenden Themenkreise:

- die Unterschiede zwischen dem Begriff der gerichteten Strecken und der geometrischen Vektoren;

- die Verbindung des mathematischen Vektors mit dem physischen Vektor als Größe;

- der Weg des axiomatischen Aufbaus.

Megyesi und seine Mitarbeiter (1974, 1982), sowie Varsics (1992) haben die Zusammenfassung und die Analyse dieses Problemkreises aus der Fachliteratur vorgenommen. Die folgende Analyse des Verhandlungskontextes beruht vor Allem auf diesen Studien.

\section{Verhandlungskontexte des Vektorbegriffs}

Die folgende mathematikdidaktische Anwendung der formalen Analyse geht von der Objektmenge der verschiedenen Vektormodelle und von der Eigenschaftsmenge einiger mathematikdidaktischer Untersuchungsaspekte aus. 


$$
\text { "fatalin" - 2005/6/30 - 16:51 - page } 4-\# 4
$$

Nach der Fachliteraturen können die folgenden elementargeometrischen Modelle des abstrakten Vektorbegriffs zusammengesammelt werden: geometrischer Vektor; Verschiebung; Ortsvektor und centroaffine Transformation mit seinem Unterraum, als geometrische Repräsentation von linearen Funktionen. In allgemeinem kommen die folgenden algebraischen Modelle vor: die geordneten $n$-Tuppel, das Polynom mit seinen Unterräumen und die Matrix. (Die weiteren Vektorinterpretationen sind die verschiedenen Funktionsräume mit unendlicher Dimension und der Begriff des Tangentvektors als allgemeinstes geometrisches Modell.) Noch in vielfältigem Gebiet (z.B. die Farbenvektoren nach Grassmann, oder das physische Vektorfeld und verschiedene logistische Anwendungen) können Vektormodelle gefunden werden, aber in diesem Artikel wird nur die mathematikdidaktische Begründung untersucht.

Die Fachliteratur hebt mehrere Aspekte bei der Vektormodelluntersuchung hervor. Einige von diesen Aspekten verknüpfen sich unmittelbar mit dem mathematischen Charakter des abstrakten Vektorbegriffs. Die axiomatische Definition des Vektorraums involviert den abstrakten Vektorbegriff (Halmos, 1974). Aus diesem Axiomensystem können die Haupteigenschaften des abstrakten Vektors festgesetzt werden. In allen Vektorräumen sind zwei Operationen gegeben: die Vektorsumme und das Multiplizieren eines Vektors durch einen Skalar. Diese Operationseigenschaften enthält das Axiomensystem und diese Postulate werden von allen Vektormodellen natürlich erfüllt.

Aus didaktischer Sicht ist es wichtig, dass beide Operationen eine unmittelbare Deutbarkeit haben. In allgemeinem ist die Deutung der Vektorsumme in elementaren Vektormodellen einfach, zwei Vektoren können glattweg addiert werden (z.B. mit der Parallelogrammregel, mit der Komposition der Verschiebungen, oder nach der Koordinatensumme), nur die geometrischen Vektoren bringen ein Problem mit: die geometrischen Vektoren wurden mit der Klassenbildung aufgebaut, so dass die Unabhängigkeit von Repräsentanten bei den Vektorsummen und beim Multiplizieren eines Vektors durch einen Skalar bewiesen werden muss. Das Multiplizieren eines Vektors durch einen Skalar ist eine ungewöhnliche Operation, weil die Operanden zu verschiedene Mengen enthalten. Diese Operation kann in elementargeometrischen Modellen einfach eingeführt werden, wenn die Vektornorm in Anspruch genommen wird, sonst muss die Menge der Skalare (ein Körper) auch aufgebaut werden. (In verschiedenen Fällen können die rationellen, reellen, komplexen Zahlen und auch die endlichen Körper die Rolle der Skalarmenge spielen.) 


$$
\text { "fatalin" - 2005/6/30 - 16:51 - page } 5-\# 5
$$

Der abstrakte Vektor hat keine Länge, aber einige Vektormodelle bringen selbstverständlich die Vektorlänge mit. (In den elementargeometrischen Vektormodellen kommt die Vektorlänge natürlicher Weise vor.) Die Vektorlänge, die Vektornorm führt eine Metrik in den Raum ein, aber sie ist keine relevante Eigenschaft des Vektorraums. Einige algebraische Vektormodelle bringen selbstverständlich den Koordinatenbegriff mit. Dieser bedeutet die Existenz einer natürlichen Basis, aber der abstrakte Vektorraum hat keine. In allen Vektormodellen können die Begriffe der linearen Unabhängigkeit und das Generatorsystem interpretiert werden. Mit Hilfe dieser Begriffe können die Elemente des Vektorraums eindeutig dargestellt und nach den Koordinaten beschrieben werden, aber zu diesen muss man eine Basis fixieren. Die Anzahl der Elemente der Basis gibt die Dimension an. Die elementargeometrischen Vektormodelle können eindimensional, zweidimensional oder dreidimensional sein, weil sie sich aus der Ortsbestimmung in eine Gerade, in eine Ebene oder in einen Raum entfaltet haben. Der abstrakte Vektorraum hat keine solche Maximalschranke. Aus dem Augenwinkel der Verallgemeinerung ist der abstrakte Vektor bei der Begriffsbildung vorteilhaft, denn die Modellkonstruktionen haben keine Dimensionsmaximalschranke. Diese Eigenschaften der Vektormodelle (der Vektor hat eine Länge, existiert eine natürliche Basis bzw. eine obere Dimensionsschranke) sind unwesentlich für den abstrakten Vektorbegriff, aber die Erfüllungen dieser Eigenschaften können die Studenten fehlleiten. In diesem Sinne werden die Metrikunabhängigkeit, die Koordinatenunabhängigkeit und die Dimensionsunabhängigkeit des Vektormodells bevorzugt.

Die weiteren Aspekte verknüpfen sich nicht mit dem mathematischen Charakter des Vektorbegriffs, sondern sie nehmen auf einige generelle mathematikdidaktische Aspekte Rücksicht. Ein funktionelles Erfordernis ist die Verschiebbarkeit des Vektors, weil dies eine wichtige Rolle für die folgerichtigen Deutungen des physischen Vektorbegriffs, des Vektorfelds spielt (Megyesi \& Skrapits, 1974; Varsics, 1992). Ein Vektormodell bekommt einen Vorteil aus didaktischer Sicht, wenn die Gleichheit des Vektors sich nach der Identität der Objekte erklärt (Reimann, 1971 und Czapáry, 1996). Die Begriffsidentizifierung ist ein weiterer, sowohl aus erkenntnistheoretischer, als auch mathematischer und didaktischer Sicht wichtiger Aspekt beim Begriffsaufbau (Ruzsa, 1971).

Eine von den didaktischen Aspekten im Lehrplanaufbau ist, dass die begründeten Modelle des abstrakten Vektorbegriffs zum Grundstoff gehören. Dieses Attribut hängt vom Lehrstoffes ab, aber man muss seine Bedeutung betonen, weil nur ein solcher Begriff als Grundkenntnis der Abstraktion funktionieren kann, die wir in das vordere Begriffsnetz schon entsprechend eingebaut haben. Zurzeit hat
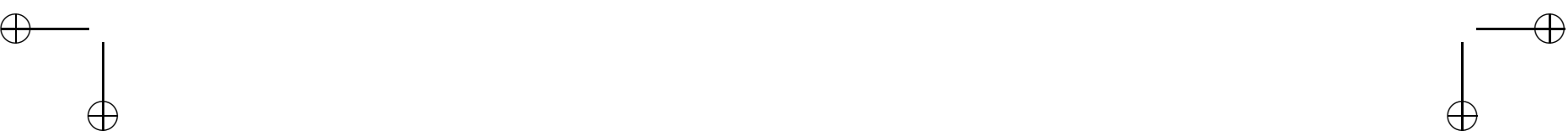
der Grundstoff in Ungarn und in einigen osteuropäischen Ländern keine Matrix, daher kann die Matrix nicht eine Rolle der Grundkenntnisse spielen. In diesen Ländern können nur die geordneten $n$-Tuppel und das Polynom als algebraische Vektormodelle funktionieren. In anderen Ländern (z.B. in Frankreich) gehört die Matrix zum Lernstoff, dort kommt die Matrix natürlich als begründende Vektorinterpretation vor. In Ungarn gehört die Matrix nur in speziellen Klassen zum Lernstoff (Fried, 1986).

Im Folgenden wird die Relationstabelle (Tabelle 1.) die Rolle des mathematischen Modells der Verhandlungskontexte des Vektorbegriffs spielen.

Tabelle 1. Die Kontexttabelle des Vektorbegriffs in den metrischen Fällen

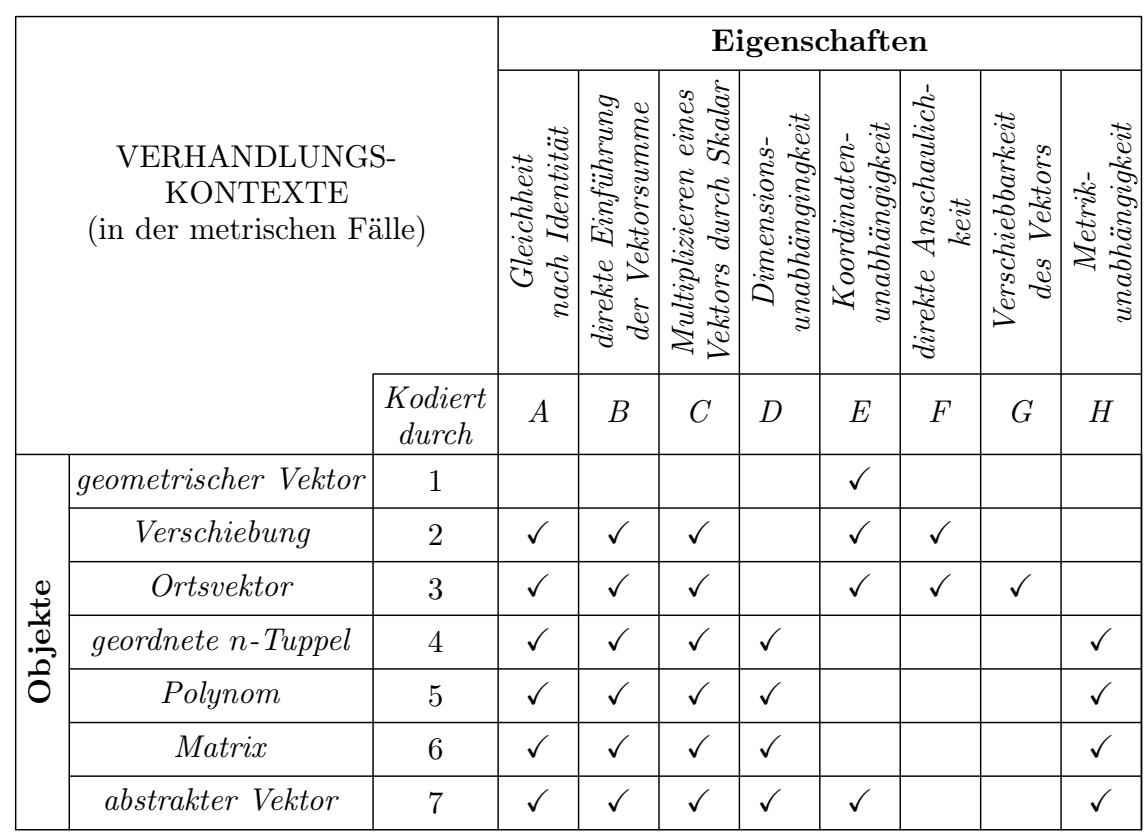

Aus der Kontexttabelle können die Eigenschaften, die ein konkretes Vektormodell besitzt oder nicht besitzt, gleichzeitig herausgelesen werden, und desgleichen ist es auch von allen Vektormodellen feststellbar, ob diese eine konkrete Eigenschaft erfüllen oder nicht. Der formalen Begriffsanalyse hilft die weitere Untersuchung mit den Herstellungen der Cliquen und des Galois-Graphen. 


$$
\text { "fatalin" }-2005 / 6 / 30-16: 51-\text { page } 7-\# 7
$$

\section{Die Cliquen und der Galois-Graph des Vektorbegriffs}

Eine Clique zeigt sich in der Kontexttabelle als eine maximale vollständige Teiltabelle, ein Rechteck, das in jeder Zelle ein Zeichen enthält und sich weder durch Objekte noch durch Eigenschaften erweitern lässt.

Die Cliquen der Tabelle 1. enthält die Tabelle 2. Diese Cliquen können als potentiale Begriffe dieser Verhandlungskontexte interpretiert werden. Die erste und die neunte Clique wären leeren Begriffen, weil die Erste keinen Begriffsinhalt und die Neunte keinen Begriffsumfang hat. Mehrere Cliquen können als Begriff funktionieren. Z. B. zeigt die zweite Clique, dass nur die Vektormodelle 2, 3, 4, 5, 6 und 7 die Eigenschaften $A, B$ und $C$ besitzen. Die Eigenschaft $G$ kommt nur in der siebten Clique vor, d.h. für die Deutung des physischen Vektorbegriffs, des Vektorfelds ist der Ortsvektor brauchbar, doch nur dieser Vektor kann in diesem Verhandlungsaspekt verschoben werden.

Tabelle 2. Die Cliquen der Relation

\begin{tabular}{|c|c|c|}
\hline \multicolumn{3}{|c|}{ CLIQUEN } \\
\hline$\lambda$ & $\begin{array}{c}\text { Objektmenge } \\
\text { der Clique }\end{array} \times \begin{array}{l}\text { Eigenschaftsmenge } \\
\text { der Clique }\end{array}$ & $\begin{array}{c}\text { Benennung } \\
\text { der Clique }\end{array}$ \\
\hline 1. & $\{1 ; 2 ; 3 ; 4 ; 5 ; 6 ; 7\} \times \emptyset$ & $\begin{array}{l}\text { Clique ohne Begriffsinhalt } \\
\text { (ein leeren Begriff) }\end{array}$ \\
\hline 2. & $\{2 ; 3 ; 4 ; 5 ; 6 ; 7\} \times\{\boldsymbol{A} ; \boldsymbol{B} ; \boldsymbol{C}\}$ & $\begin{array}{l}\text { Clique der relevanten } \\
\text { Eigenschaften }\end{array}$ \\
\hline 3. & $\{1 ; 2 ; 3 ; 7\} \times\{\boldsymbol{E}\}$ & $\begin{array}{l}\text { Clique der Koordinaten- } \\
\text { unabhängigkeit }\end{array}$ \\
\hline 4. & $\{4 ; 5 ; 6 ; 7\} \times\{A ; B ; C ; \boldsymbol{D} ; \boldsymbol{H}\}$ & $\begin{array}{l}\text { algebraische Begründung } \\
\text { des abstrakten Vektors }\end{array}$ \\
\hline 5 . & $\{2 ; 3 ; 7\} \times\{A ; B ; C ; E\}$ & $\begin{array}{l}\text { geometrische Begründung } \\
\text { des abstrakten Vektors }\end{array}$ \\
\hline 6. & $\{2 ; 3\} \times\{A ; B ; C ; E ; \boldsymbol{F}\}$ & Clique der Anschaulichkeit \\
\hline 7. & $\{3\} \times\{A ; B ; C ; E ; \boldsymbol{G} ; F\}$ & $\begin{array}{l}\text { Ortsvektor oder } \\
\text { Clique der Verschiebbarkeit }\end{array}$ \\
\hline 8. & $\{7\} \times\{A ; B ; C ; D ; E ; H\}$ & abstrakter Vektor \\
\hline 9 . & $\emptyset \times\{A ; B ; C ; D ; E ; F ; G ; H\}$ & $\begin{array}{l}\text { Clique ohne Begriffsumfang } \\
\text { (ein leeren Begriff) }\end{array}$ \\
\hline
\end{tabular}




$$
\text { "fatalin" - 2005/6/30 - 16:51 - page } 8-\# 8
$$

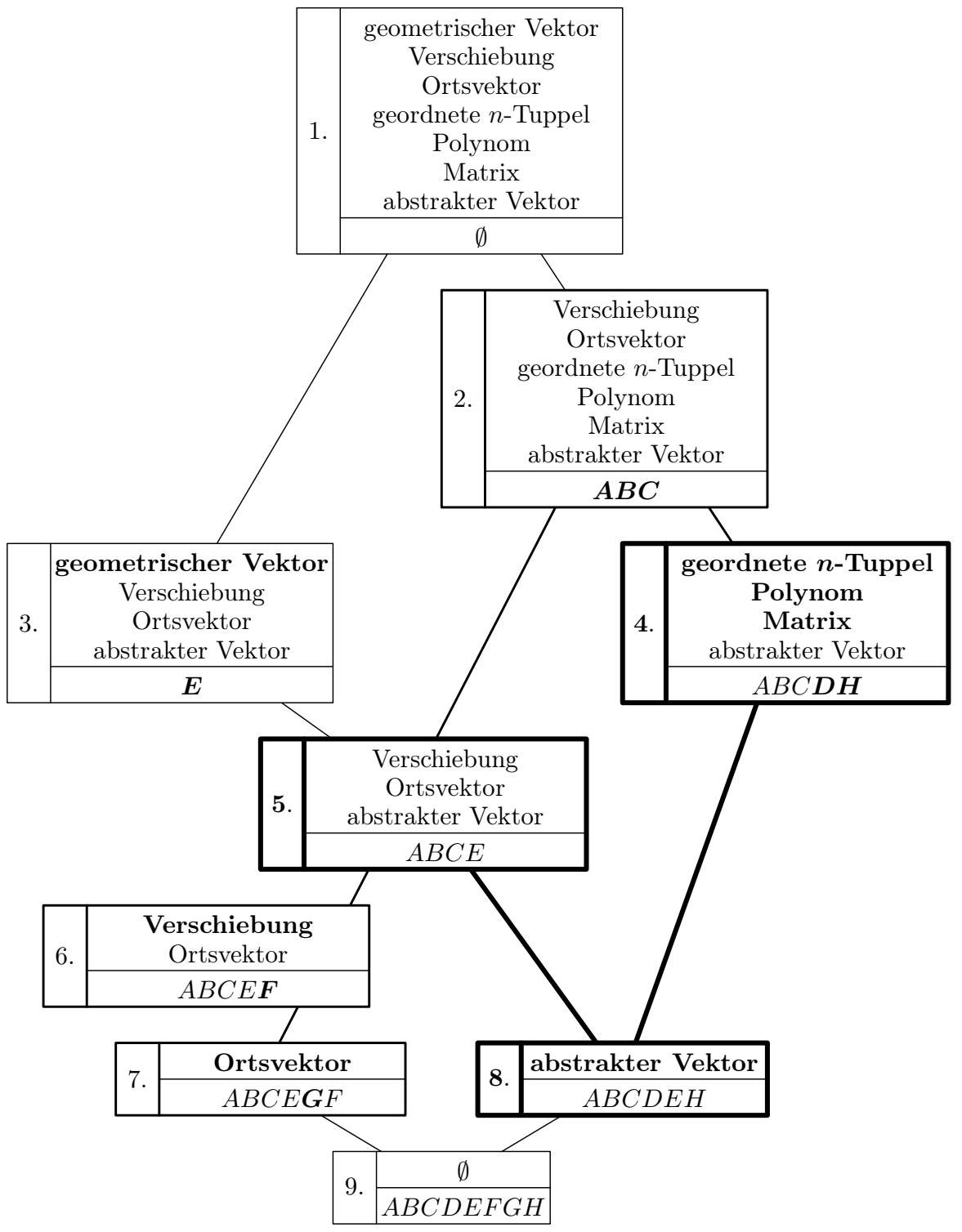

Abbildung 1. Der Galois-Graph des Vektorbegriffs im metrischen Fall

Nach der einführbaren Ordnungsrelation der Cliquen kann das Hasse-Diagramm der geordneten Menge gezeichnet werden. Der Galois-Graph des Vektorbegriffs ist in der Abbildung 1. ersichtlich. Der Aufbau dieses Galois-Graphen lässt sich - wie folgt - charakterisieren: 


$$
\text { "fatalin" - 2005/6/30 - 16:51 — page } 9-\# 9
$$

- An der unmittelbaren Begründung des abstrakten Vektorbegriffs (achte Clique) nehmen die vierte und die fünfte Clique teil.

- Der abstrakte Vektor und seine unmittelbare Begründung haben die gesamten Eigenschaften $A, B$ und $C$.

- Die relevanten Eigenschaften $(A, B, C)$ des abstrakten Vektorbegriffs werden mit der Eigenschaft $E$ nach der geometrischen Begründung und mit den Eigenschaften $D$ und $H$ nach der algebraischen Begründung ergänzt.

- Der geometrische Vektor hat keine unmittelbare Rolle in dem Aufbau des abstrakten Vektorbegriffs.

\section{Mathematikdidaktischen Konklusionen}

Dieser Galois-Graph gibt eine exakte Beschreibungsform des Vektorbegriffs, was zu den didaktischen Konklusionen verhelfen kann. Der abstrakte Vektorbegriff hat sechs Eigenschaften $(A B C D E H)$ in diesem Verhandlungskontext. Diese Eigenschaften sind wichtig aus dem mathematikdidaktischen Gesichtpunkt. Die relevanten Eigenschaften des abstrakten Vektorbegriffs in der Mathematik sind die Eigenschaften $A, B$ und $C$. Nur die zweite Clique und seine Teilcliquen haben diese Eigenschaften, daher ist die Einführung des geometrischen Vektors zum abstrakten Vektorbegriff unnötig (Varsics, 1992). Es ist bemerkenwert, dass die Grundbeschaffenheit des Galois-Graphen unverändert bleiben würde, wenn wir nur die Einführung der Operationen (unabhängig von der Methode) betrachtet hätten.

Ein Vektorbegriff wird im mathematikdidaktischen Sinn entsprechend genannt, wenn er sehr brauchbar - ohne weitere Verallgemeinerung - ist. Vor allem bekommen die linearen Kombinationen die Hauptrolle bei der Vektorsabstraktion, weil die Vektorlänge oder die natürliche Basis nur manchmal existieren. Meistens haben die mehrdimensionalen Vektorräume - z.B. in der Ökonomie eine natürliche Basis, aber keine Länge. Der dreidimensionale Farbenvektor hat keine natürliche Länge und keine natürlicher Basis. (Das Windows-System benutzt die RGB-Basis und auch die HSL-Basis.) Der Galois-Graph als Begriffsnetz zeigt, dass sowohl eine algebraische (geordnete $n$-Tuppel, Polynom und Matrix) als auch eine geometrische (Ortsvektor und Verschiebung) Interpretation an der unmittelbaren Begründung des abstrakten Vektorbegriffs teilnehmen. Zum entsprechenden abstrakten Vektorbegriff sind beide Begründungen nötig, doch die relevanten Eigenschaften $(A, B, C)$ werden mit der Koordinatenunabhängigkeit 
"fatalin" - 2005/6/30 - 16:51 — page $10-\# 10$

nach der geometrischen Begründung und mit der Dimensionsunabhängigkeit und Metrikunabhängigkeit nach der algebraischen Begründung ergänzt.

In der formalen Begriffsanalyse spielen die Kontextmanipulationen (die Bereinigung und die Reduzierung) eine wichtige Rolle, weil sie zur engen Verbindung von vollständigen Verbänden der Relation nötig sind. Unter epistemologischem Aspekt sind zwei Objekte identisch, wenn sie in jeder ihrer Eigenschaften gleich sind. Im untersuchten Verhandlungskontext sind die geordnete $n$-Tuppel, das Polynom und die Matrix voneinander nicht verschieden. Die geordneten $n$-Tuppel, das Polynom und die Matrix sind aus dem Gesichtswinkel der Erkenntnislehre in diesem Kontext gleiche Vektorinterpretationen.

In mathematikdidaktischer Anwendung ist es günstig den Vektorbegriff mit der vollständigen Relation zu untersuchen. Die Eigenschaften $B$ und $C$ (direkte Einführung der Vektorsumme und des Multiplizierens eines Vektors durch Skalar) haben den gleichen Begriffsumfang in diesem Verhandlungskontext. Auf diese Weise kann dieser identifiziert werden und seine Synthese benennen wir als ,,direkte Einführung der Vektoroperationen". Ohne diese Kontextbereinigung können die metrischen und affinen Fälle der geometrischen Vektorinterpretationen untersucht werden. Der abstrakte Vektorbegriff hat keine metrische Eigenschaft. Die geometrischen Vektormodelle können als affiner Begriff auch nur nach der Inzidenzrelation des axiomatischen Systems aufgebaut werden (Emil Molnár, 1972). Die Theoreme von Desargues und Pappos-Pascal bekommen die Hauptrolle im affinen Aufbau (Hilbert \& Cohn-Vossen, 1932). Obwohl diese Themen keine Rolle in der Mittelschule (Sekundarstufe) haben, ist es doch ein wichtiger Aspekt der Ausbildung des Vektorbegriffs, dass die vorderen Kenntnisse bei der späteren Begriffsverallgemeinerung nicht hinderlich sind. Die Tabelle 3. enthält das Relationsmodell des affinen Kontexts. Es ist ersichtlich, dass nur die Eigenschaften $C$ (Multiplizierens eines Vektors durch einen Skalar) und H (Metrikunabhängigkeit) einzusetzen sind, woraus sich das Folgende ergibt: die Grundbeschaffenheit des Galois-Graphen - das Begriffsnetz - bleibt unverändert. Die Erhaltung des Begriffsnetzes hilft der Verallgemeinerung, doch das gelernte Schema wird nicht modifiziert.

Die Schülertätigkeit kann mit entsprechenden Aufgabenserien gelenkt werden. Die strukturelle Bewertung der Aufgabenserien und deren Schülerlösungen können auch mit diesem Relationsmittel gemacht werden, aber dieses begründet sich in der Elaboration des angezielten Wissensnetzes (Fatalin 2003b). Aus diesem Gesichtswinkel ist dieses Begriffsnetz-Modell auch vorteilhaft. 
Tabelle 3. Die Kontexttabelle des affinen Verhandlungskontexts

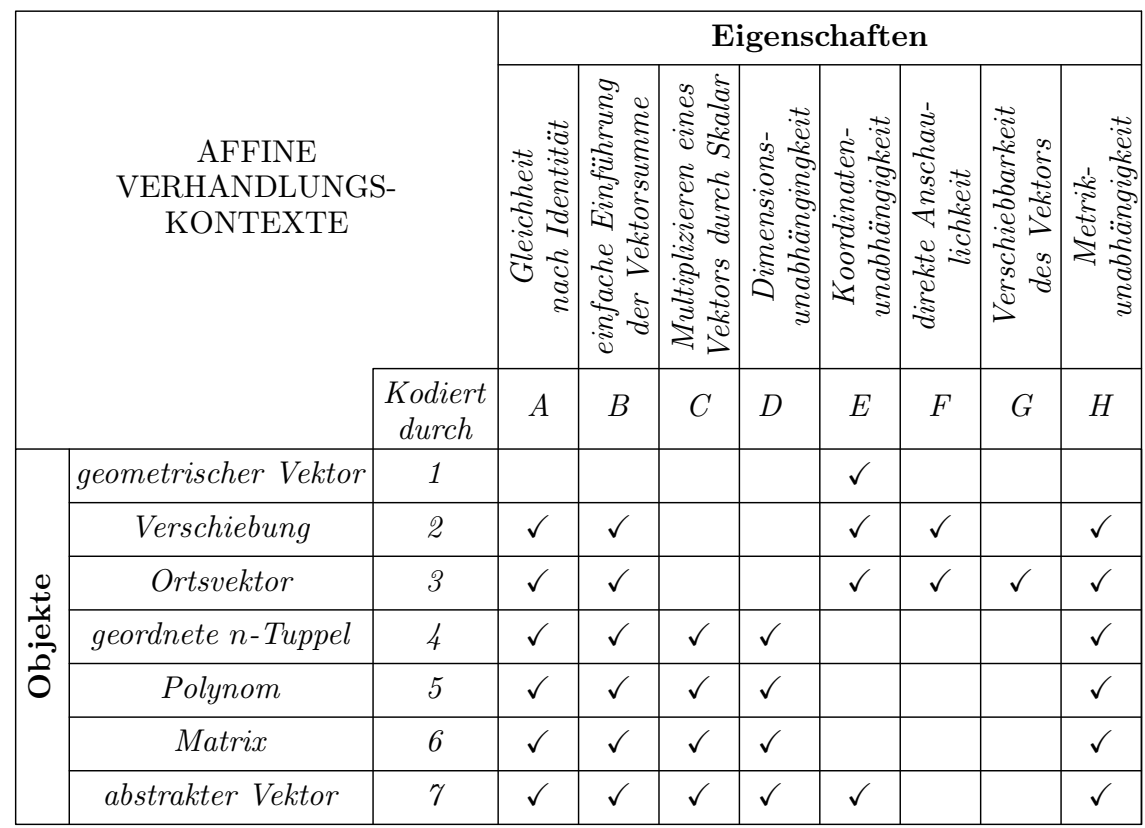

\section{Literatur}

[1] A. Ambrus, Bevezetés a matematikadidaktikába, Eötvös Kiadó, Budapest, 1995.

[2] W. G. Boltjanszkij and I. M. Jaglom, Энииклопедия элэмэнтарной математики, Гостехиздат, Москва, 1963, (deutsch: Vektoren und ihre Anwendungen in: Enzyklopädie der Elementarmathematik IV. der Geometrie) VEB Deutscher Verlag der Wissenschaften Berlin 1980. 297-302.

[3] E. Czapáry, Tanári kézikönyv a szakközépiskolák I. és II. osztályos matematika tananyagának tanításához, Nemzeti Tankönyvkiadó, Budapest, 1996.

[4] L. Fatalin, Strukturelle Analyse des Lernstoffes, (K. J. Parisot \& É. Vásárhelyi, eds.), Neue Sichtweisen in der Didaktik der Mathematik. Abakus, Salzburg, 2003, $45-56$.

[5] L. Fatalin, Strukturelle Analyse von Aufgabenserien und deren Schülerlösungen, (K. J. Parisot \& É. Vásárhelyi, eds.), Neue Sichtweisen in der Didaktik der Mathematik. Abakus, Salzburg, 2003, 59-78.

[6] E. Fried, Lineáris algebra a speciális matematikai osztályok számára, Tankönyvkiadó, Budapest, 1986.

[7] B. Ganter and R. Wille, Formale Begriffsanalyse, Springer, Berlin, 1996.

[8] P. R. Halmos, Finite-dimensional Vector Spaces, Springer, New York, 1974. 


$$
\text { "fatalin" — 2005/6/30 - 16:51 — page } 12-\# 12
$$

[9] D. Hilbert and S. Cohn-Vossen, Anschauliche Geometrie, in: Grundlehren der mathematischen Wissenschaften XXXVII, Springer, Berlin - Heidelberg - New York, 1932.

[10] L. Megyesi and L. Skrapits, Über die Vektorgrösse..., (ungarisch: A vektormenynyiségek és a vektor fogalmáról), in: ELTE TTK Szakmódszertani közlemények, Budapest 7 (1974), 176-203.

[11] E. Molnár, Geometrische Axiomatik und Relativitätstheorie, (ungarisch: Geometriai axiomatika és relativitáselmélet), in: ELTE TTK Szakmódszertani közlemények, Budapest 5 (1972), 72-112.

[12] J. Peller and L. Megyesi, Függvények elemi vizsgálata. Vektorok, A tanulók matematikai tevékenységének tervezése és irányítása a középiskolában III., Tankönyvkiadó, Budapest, 1982.

[13] I. Reiman, Vektorok a geometriában, Szakköri feladatgyüjtemény, Tankönyvkiadó, Budapest, 1971.

[14] I. Ruzsa, A matematika néhány filozófiai problémájáról, Tankönyvkiadó, Budapest, 1971.

[15] V. Takács, A Galois-gráf pedagógiai alkalmazásai, Iskolakultúra, Pécs (2000).

[16] Z. Varsics, Aufbau des Vektorbegriffs, (ungarisch: A vektorfogalom kialakítása), Iskolakultúra Jg. 2, no. 3 (1992), 2-7.

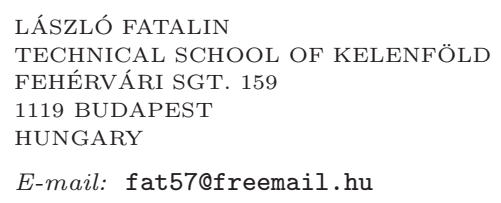

(Received September, 2004) 